\title{
Machine-Learning-Directed Navigation of Synthetic Design Space: A Statistical Learning Approach to Controlling the Synthesis of Perovskite Halide Nanoplatelets in the Quantum- Confined Regime
}

Erick J. Braham, ${ }^{\dagger, \S \# ~ J u n s a n g ~ C h o, ~}{ }^{+, \S \#}$ Kristel M. Forlano, ${ }^{+}$David F. Watson, II Raymundo Arròyave,,$^{\S}$ Sarbajit Banerjee $^{\dagger, \S *}$

${ }^{\dagger}$ Department of Chemistry, Texas A\&M University, College Station, TX, 77843-3255, USA

$\S$ Department of Materials Science and Engineering, Texas A\&M University, College Station, TX 778433255, USA

"Department of Chemistry, University at Buffalo, The State University of New York, Buffalo, New York 14260-3000, USA

\#these authors contributed equally to this work 


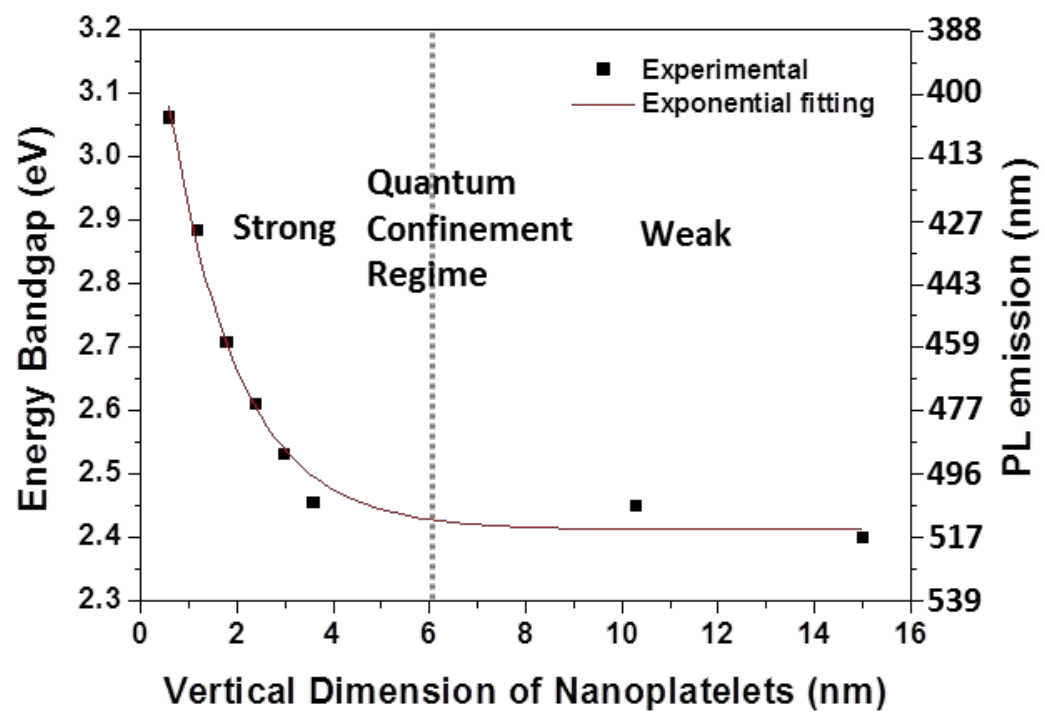

Figure S1. PL emission wavelength (or energy bandgap) of 2D CsPbBr 3 nanoplatelets derived from single-particle measurements plotted as a function of the vertical dimension of the nanoplatelets.

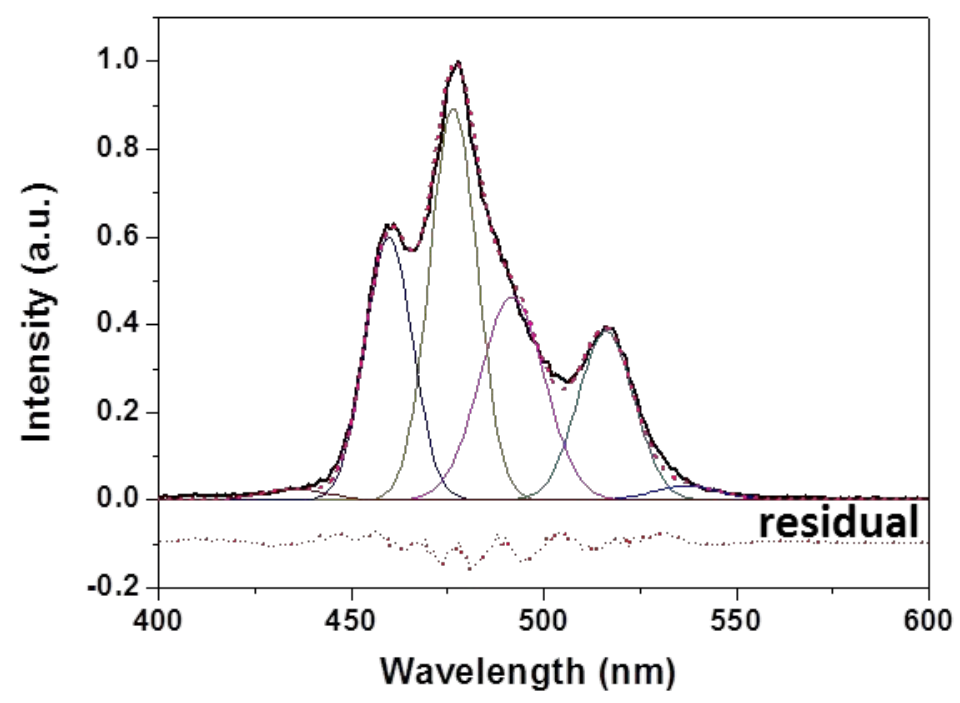

Figure S2. Deconvolution of $\mathrm{PL}$ emission spectra with multiple Gaussian fitting for $\mathrm{CsPbBr}_{3}$ nanoplatelets obtained at the reaction condition of using $\mathrm{C} 12$ with 1:20 at $150{ }^{\circ} \mathrm{C}$. The dotted line at the bottom of the plot represents the residual of the fit. 


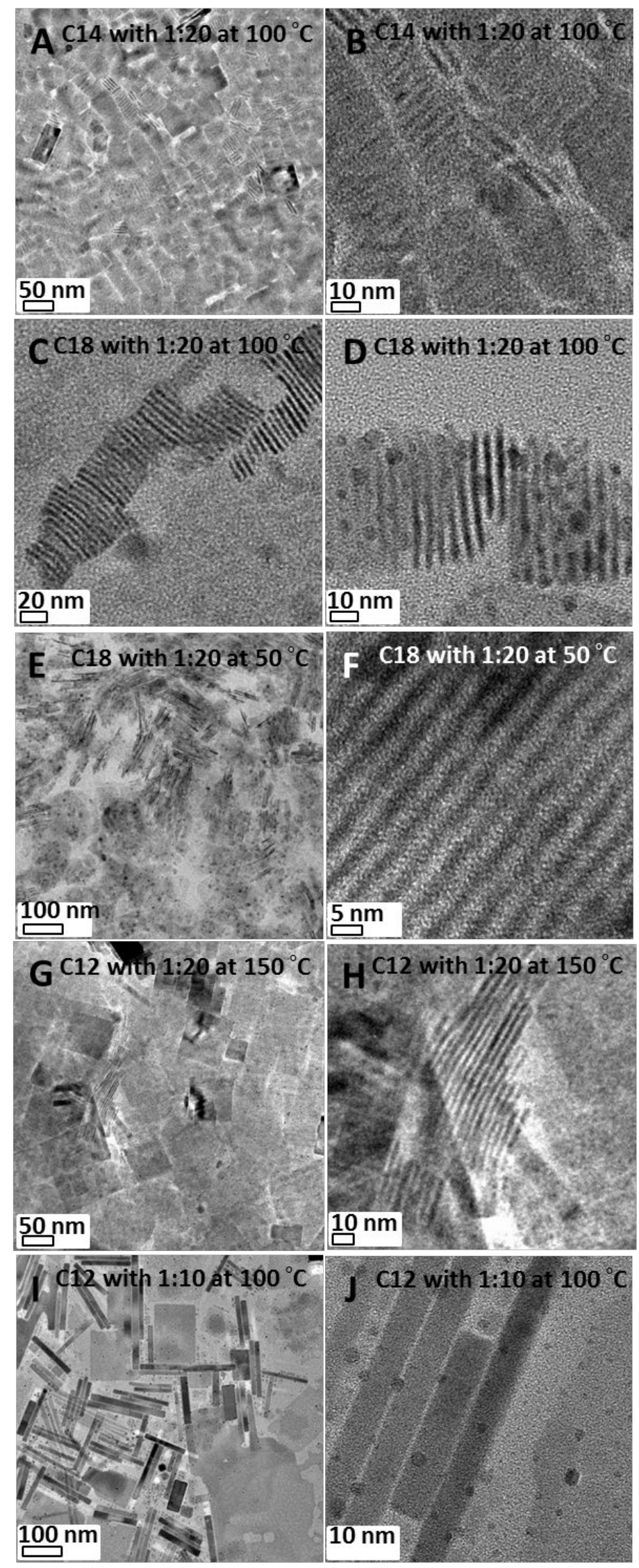

Figure S3. TEM images of 2D CsPbBr 3 nanoplatelets grown under different reaction conditions: $A, B$ ) C14 with $1: 20$ at $\left.100^{\circ} \mathrm{C} ; \mathrm{C}, \mathrm{D}\right) \mathrm{C} 18$ with $1: 20$ at $\left.100^{\circ} \mathrm{C} ; \mathrm{E}, \mathrm{F}\right) \mathrm{C} 18$ with $1: 20$ at $\left.50^{\circ} \mathrm{C} ; \mathrm{G}, \mathrm{H}\right) \mathrm{C} 12$ with $1: 20$ at $\left.150^{\circ} \mathrm{C} ; \mathrm{I}, \mathrm{J}\right) \mathrm{C} 12$ with $1: 10$ at $100^{\circ} \mathrm{C}$. 


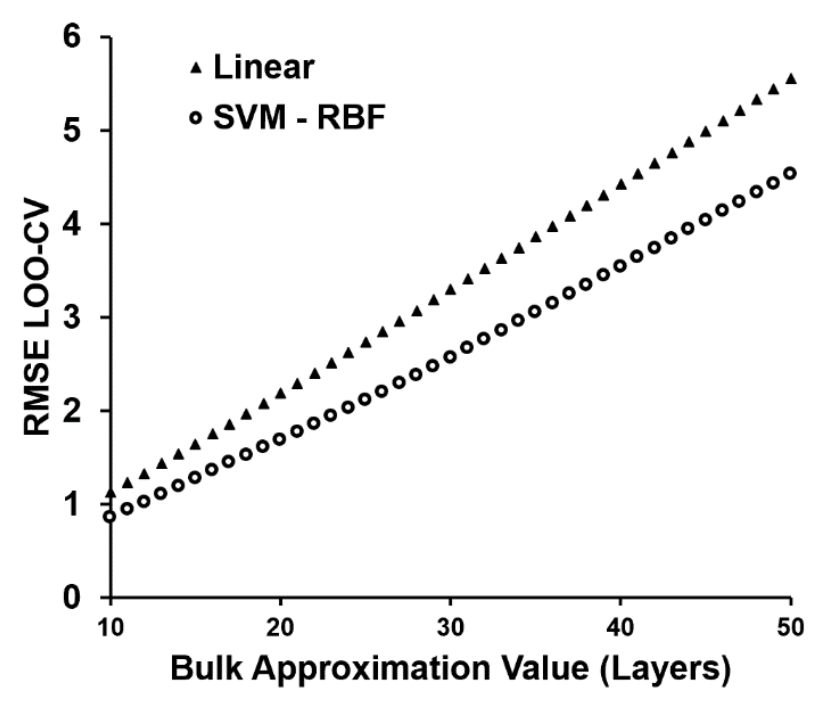

Figure S4: Plot of leave-one-out cross-validation RMSE-CV as a function of the bulk thickness approximation for linear and SVM with a radial basis function kernel models using diffusion coefficient as the alkylamine descriptor.

Table S1. Deconvolution of PL emission spectra for an initial sampling of multivariate reaction space (40 samples). Multi-peak Gaussian fitting has been used to determine the fractional populations for different layer thicknesses ( $n=1,2,3,4,5,6$, and bulk). Grey shading indicates samples with $<5 \%$ bulk PL emission intensity. Blue shading indicates samples with $>50 \%$ bulk PL emission intensity.

\begin{tabular}{|c|c|c|c|c|c|c|c|c|c|c|}
\hline Entry & $\begin{array}{l}\text { Alkylami } \\
\text { ne Chain- } \\
\text { length } \\
\text { (Cn) }\end{array}$ & $T\left({ }^{\circ} \mathrm{C}\right)$ & $\begin{array}{c}\text { Conc. } \\
(x)\end{array}$ & $n=2$ & $n=3$ & $n=4$ & $n=5$ & $n=6$ & $\begin{array}{c}n= \\
\text { bulk }\end{array}$ & $n_{\text {avg. }}$ \\
\hline 1 & 4 & 50 & $1: 20$ & - & 0.204 & 0.198 & 0.289 & - & 0.309 & 5.95 \\
\hline 2 & 4 & 100 & $1: 20$ & - & - & 0.060 & 0.091 & 0.094 & 0.755 & 8.81 \\
\hline 3 & 4 & 100 & $1: 5$ & - & - & - & - & - & 1.000 & 10.0 \\
\hline 4 & 4 & 100 & $1: 10$ & - & - & - & - & - & 1.000 & 10.0 \\
\hline 5 & 4 & 100 & $1: 20$ & - & - & 0.035 & 0.125 & 0.170 & 0.671 & 8.49 \\
\hline 6 & 4 & 100 & $1: 30$ & - & 0.045 & 0.060 & 0.087 & 0.146 & 0.661 & 8.30 \\
\hline 7 & 4 & 100 & $1: 40$ & - & - & - & - & 0.191 & 0.809 & 9.23 \\
\hline 8 & 4 & 150 & $1: 20$ & - & 0.023 & 0.028 & 0.017 & - & 0.932 & 9.59 \\
\hline 9 & 8 & 50 & $1: 20$ & 0.021 & 0.508 & 0.177 & 0.061 & - & 0.232 & 4.84 \\
\hline 10 & 8 & 100 & $1: 20$ & 0.009 & 0.297 & 0.227 & 0.113 & - & 0.354 & 5.92 \\
\hline 11 & 8 & 100 & $1: 5$ & - & - & - & 0.071 & 0.243 & 0.686 & 8.67 \\
\hline 12 & 8 & 100 & $1: 10$ & 0.011 & 0.017 & 0.109 & 0.225 & - & 0.638 & 8.01 \\
\hline 13 & 8 & 100 & $1: 20$ & 0.015 & 0.326 & 0.194 & 0.080 & 0.071 & 0.314 & 5.75 \\
\hline 14 & 8 & 100 & $1: 30$ & 0.456 & 0.383 & 0.090 & 0.023 & 0.017 & 0.032 & 2.95 \\
\hline 15 & 8 & 100 & $1: 40$ & - & 0.514 & 0.332 & 0.078 & 0.076 & - & 3.71 \\
\hline 16 & 8 & 150 & $1: 20$ & - & - & - & - & 0.053 & 0.947 & 9.77 \\
\hline 17 & 12 & 50 & $1: 20$ & 0.412 & 0.527 & 0.025 & 0.017 & - & 0.019 & 2.78 \\
\hline 18 & 12 & 100 & $1: 20$ & 0.047 & 0.493 & 0.246 & 0.119 & - & 0.096 & 4.11 \\
\hline 19 & 12 & 100 & $1: 5$ & 0.025 & 0.015 & 0.035 & 0.055 & - & 0.870 & 9.21 \\
\hline 20 & 12 & 100 & $1: 10$ & - & 0.015 & 0.179 & 0.141 & 0.047 & 0.618 & 7.92 \\
\hline
\end{tabular}




\begin{tabular}{|l|c|c|c|c|c|c|c|c|c|c|}
\hline 21 & 12 & 100 & $1: 20$ & 0.460 & 0.443 & 0.050 & 0.027 & 0.008 & 0.012 & 2.75 \\
\hline 22 & 12 & 100 & $1: 30$ & 0.387 & 0.486 & 0.081 & 0.025 & 0.015 & 0.006 & 2.83 \\
\hline 23 & 12 & 100 & $1: 40$ & 0.030 & 0.661 & 0.190 & 0.070 & 0.023 & 0.025 & 3.56 \\
\hline 24 & 12 & 150 & $1: 20$ & 0.013 & 0.221 & 0.351 & 0.229 & - & 0.186 & 5.10 \\
\hline 25 & 14 & 50 & $1: 20$ & 0.108 & 0.746 & 0.083 & 0.037 & 0.026 & - & 3.10 \\
\hline 26 & 14 & 100 & $1: 20$ & 0.357 & 0.524 & 0.079 & 0.02 & 0.019 & - & 2.81 \\
\hline 27 & 14 & 100 & $1: 5$ & 0.024 & 0.052 & 0.105 & 0.087 & 0.073 & 0.659 & 8.09 \\
\hline 28 & 14 & 100 & $1: 10$ & - & 0.253 & 0.426 & 0.193 & 0.055 & 0.073 & 4.48 \\
\hline 29 & 14 & 100 & $1: 20$ & 0.358 & 0.524 & 0.079 & 0.021 & 0.019 & - & 2.82 \\
\hline 30 & 14 & 100 & $1: 30$ & 0.531 & 0.378 & 0.054 & 0.018 & 0.019 & - & 2.62 \\
\hline 31 & 14 & 100 & $1: 40$ & 0.098 & 0.657 & 0.184 & 0.039 & 0.023 & - & 3.23 \\
\hline 32 & 14 & 150 & $1: 20$ & 0.010 & 0.385 & 0.312 & 0.096 & 0.030 & 0.168 & 4.76 \\
\hline 33 & 18 & 50 & $1: 20$ & 0.012 & 0.684 & 0.179 & 0.107 & - & 0.017 & 3.50 \\
\hline 34 & 18 & 100 & $1: 20$ & 0.063 & 0.589 & 0.234 & 0.076 & 0.039 & - & 3.44 \\
\hline 35 & 18 & 100 & $1: 5$ & 0.019 & 0.046 & 0.101 & 0.096 & - & 0.738 & 8.43 \\
\hline 36 & 18 & 100 & $1: 10$ & 0.017 & 0.629 & 0.185 & 0.078 & 0.015 & 0.076 & 3.91 \\
\hline 37 & 18 & 100 & $1: 20$ & 0.035 & 0.656 & 0.125 & 0.132 & 0.053 & - & 3.52 \\
\hline 38 & 18 & 100 & $1: 30$ & 0.128 & 0.692 & 0.124 & 0.038 & 0.018 & - & 3.12 \\
\hline 39 & 18 & 100 & $1: 40$ & - & 0.484 & 0.245 & 0.151 & - & 0.121 & 4.40 \\
\hline 40 & 18 & 150 & $1: 20$ & - & 0.589 & 0.221 & 0.097 & 0.044 & 0.044 & 3.89 \\
\hline
\end{tabular}

Table S2. Deconvolution of PL emission spectra for additional random sampling of multivariate reaction space (34 samples) performed to expand the dataset to a viable size outside of the original experimental vectors. Multi-peak Gaussian fitting has been used to determine the fractional populations for different layer thicknesses ( $n=1,2,3,4,5,6$, and bulk). Grey shading indicates samples with $<5 \%$ bulk PL emission intensity. Blue shading indicates samples with $>50 \%$ bulk PL emission intensity.

\begin{tabular}{|c|c|c|c|c|c|c|c|c|c|c|}
\hline Entry & $\begin{array}{c}\text { Chain } \\
\text { length } \\
(\mathrm{C} n)\end{array}$ & $T\left({ }^{\circ} \mathrm{C}\right)$ & $\begin{array}{c}\text { Conc. } \\
(x)\end{array}$ & $n=2$ & $n=3$ & $n=4$ & $n=5$ & $n=6$ & $\begin{array}{c}n= \\
\text { bulk }\end{array}$ & $n_{\text {avg. }}$ \\
\hline 1 & 4 & 70 & $1: 35$ & - & 0.031 & 0.107 & 0.169 & 0.482 & 0.210 & 6.36 \\
\hline 2 & 5 & 50 & $1: 30$ & - & 0.248 & 0.172 & 0.221 & 0.322 & 0.037 & 4.84 \\
\hline 3 & 5 & 60 & $1: 35$ & - & 0.118 & 0.124 & 0.404 & 0.175 & 0.179 & 5.71 \\
\hline 4 & 5 & 110 & $1: 40$ & - & - & 0.019 & 0.205 & 0.183 & 0.594 & 8.13 \\
\hline 5 & 5 & 150 & $1: 20$ & - & - & - & - & 0.040 & 0.961 & 9.84 \\
\hline 6 & 6 & 110 & $1: 40$ & 0.012 & 0.195 & 0.116 & 0.211 & 0.089 & 0.376 & 6.43 \\
\hline 7 & 7 & 80 & $1: 30$ & 0.110 & 0.700 & 0.110 & 0.030 & 0.030 & 0.030 & 3.33 \\
\hline 8 & 7 & 80 & $1: 20$ & - & 0.146 & 0.192 & 0.083 & 0.234 & 0.345 & 6.47 \\
\hline 9 & 9 & 50 & $1: 40$ & 0.032 & 0.599 & 0.249 & 0.078 & 0.028 & 0.014 & 3.55 \\
\hline 10 & 9 & 70 & $1: 25$ & - & 0.743 & 0.123 & 0.082 & 0.037 & 0.015 & 3.51 \\
\hline 11 & 9 & 80 & $1: 40$ & - & 0.479 & 0.288 & 0.111 & 0.063 & 0.059 & 4.11 \\
\hline 12 & 9 & 90 & $1: 40$ & 0.035 & 0.600 & 0.167 & 0.131 & 0.040 & 0.027 & 3.70 \\
\hline 13 & 12 & 110 & $1: 30$ & 0.112 & 0.564 & 0.117 & 0.114 & 0.060 & 0.032 & 3.64 \\
\hline 14 & 14 & 50 & $1: 25$ & 0.065 & 0.754 & 0.093 & 0.043 & 0.029 & 0.016 & 3.31 \\
\hline 15 & 14 & 70 & $1: 25$ & 0.136 & 0.665 & 0.151 & 0.027 & 0.020 & - & 3.13 \\
\hline
\end{tabular}




\begin{tabular}{|c|c|c|c|c|c|c|c|c|c|c|}
\hline 16 & 14 & 80 & $1: 20$ & 0.020 & 0.720 & 0.200 & 0.030 & 0.030 & - & 3.33 \\
\hline 17 & 14 & 120 & $1: 30$ & 0.030 & 0.341 & 0.157 & 0.135 & 0.155 & 0.181 & 5.13 \\
\hline 18 & 14 & 120 & $1: 40$ & - & 0.338 & 0.187 & 0.069 & 0.119 & 0.287 & 5.69 \\
\hline 19 & 14 & 150 & $1: 30$ & 0.040 & 0.224 & 0.170 & 0.173 & 0.059 & 0.335 & 6.00 \\
\hline 20 & 14 & 150 & $1: 40$ & 0.046 & 0.169 & 0.276 & 0.188 & 0.118 & 0.203 & 5.38 \\
\hline 21 & 16 & 70 & $1: 40$ & 0.052 & 0.398 & 0.161 & 0.274 & 0.089 & 0.026 & 4.10 \\
\hline 22 & 16 & 90 & $1: 40$ & 0.115 & 0.527 & 0.084 & 0.033 & 0.121 & 0.119 & 4.24 \\
\hline 23 & 16 & 100 & $1: 35$ & 0.029 & 0.107 & 0.089 & 0.072 & 0.646 & 0.057 & 5.54 \\
\hline 24 & 16 & 120 & $1: 5$ & - & - & - & 0.05 & - & 0.949 & 9.74 \\
\hline 25 & 18 & 50 & $1: 15$ & 0.016 & 0.503 & 0.306 & 0.133 & 0.033 & 0.009 & 3.72 \\
\hline 26 & 18 & 50 & $1: 10$ & 0.021 & 0.217 & 0.353 & 0.202 & 0.136 & 0.071 & 4.64 \\
\hline 27 & 18 & 60 & $1: 30$ & 0.035 & 0.529 & 0.270 & 0.104 & 0.048 & 0.014 & 3.69 \\
\hline 28 & 18 & 60 & $1: 20$ & 0.052 & 0.529 & 0.259 & 0.113 & 0.033 & 0.014 & 3.63 \\
\hline 29 & 18 & 70 & $1: 20$ & 0.060 & 0.512 & 0.205 & 0.140 & 0.054 & 0.029 & 3.79 \\
\hline 30 & 18 & 100 & $1: 25$ & 0.025 & 0.306 & 0.339 & 0.230 & 0.073 & 0.026 & 4.18 \\
\hline 31 & 18 & 110 & $1: 25$ & 0.021 & 0.271 & 0.293 & 0.143 & 0.149 & 0.125 & 4.87 \\
\hline 32 & 18 & 120 & $1: 10$ & - & - & 0.05 & 0.11 & 0.76 & 0.08 & 6.09 \\
\hline 33 & 18 & 130 & $1: 40$ & 0.036 & 0.126 & 0.133 & 0.247 & 0.326 & 0.132 & 5.49 \\
\hline 34 & 18 & 140 & $1: 15$ & 0.012 & 0.480 & 0.256 & 0.109 & 0.092 & 0.051 & 4.09 \\
\hline
\end{tabular}

Table S3. Descriptor values for the alkylamines used for t-test and wrapper evaluation.

\begin{tabular}{|c|c|c|c|c|c|c|c|c|c|c|c|c|}
\hline \begin{tabular}{|l|} 
Chain \\
length
\end{tabular} & \begin{tabular}{|l|} 
Molar \\
mass \\
$(\mathrm{g} / \mathrm{mol})^{1}$
\end{tabular} & \begin{tabular}{|l|} 
Boiling \\
point \\
$\left({ }^{\circ} \mathrm{C}\right)^{2}$
\end{tabular} & $\begin{array}{l}\text { Enthalpy of } \\
\text { vaporization } \\
(\mathrm{kJ} / \mathrm{mol})^{2}\end{array}$ & \begin{tabular}{|l|} 
Density \\
$(\mathrm{g} / \mathrm{mL})^{1}$
\end{tabular} & $\mathrm{pKa}^{1}$ & $\begin{array}{l}\text { Diffusion } \\
\text { coefficient } \\
20^{\circ} \mathrm{C} \\
\left(\mathrm{m}^{2} / \mathrm{s} \times 10^{-}\right. \\
10)^{3}\end{array}$ & $\begin{array}{l}\Delta G \\
\text { agglomeration } \\
100^{\circ} \mathrm{C} \\
(\mathrm{kJ} / \mathrm{mol})^{4}\end{array}$ & $\begin{array}{l}\text { Dipole } \\
\text { moment } \\
(D)^{5}\end{array}$ & $\begin{array}{l}\text { Polarizability } \\
\left(\AA^{3}\right)^{2}\end{array}$ & \begin{tabular}{|l|} 
Melting \\
point \\
$\left({ }^{\circ} \mathrm{C}\right)^{1}$
\end{tabular} & $\begin{array}{l}{ }^{1} \mathrm{H}-\mathrm{NMR} \\
\text { shift } \\
\text { (terminal } \\
\left.\mathrm{CH}_{3}\right) \\
(\mathrm{ppm})^{6} \\
\end{array}$ & $\begin{array}{l}{ }^{1} \mathrm{H}-\mathrm{NMR} \\
\text { shift } \\
\left(-\mathrm{NH}_{2}\right) \\
(\mathrm{ppm})^{6}\end{array}$ \\
\hline 4 & 73.137 & 77.3 & 31.8 & 0.74 & 10.60 & 1.177 & 6.222 & 0.742 & 9.6 & 224 & 0.92 & 1.77 \\
\hline 5 & 87.163 & 105.5 & 34.0 & 0.755 & 10.63 & 1.147 & 1.740 & 0.723 & 11.4 & 218.15 & 0.91 & 1.12 \\
\hline 6 & 101.19 & 131.8 & 36.5 & 0.77 & 10.56 & 1.080 & -2.742 & 1.029 & 13.2 & 249.8 & 0.9 & 1.17 \\
\hline 7 & 115.217 & 156.4 & 39.3 & 0.777 & 10.67 & 0.991 & -7.225 & 0.837 & 15.1 & 250.15 & 0.89 & 1.4 \\
\hline 8 & 129.243 & 179.4 & 41.6 & 0.782 & 10.65 & 0.979 & -11.707 & 1.125 & 16.9 & 272.15 & 0.881 & 1.69 \\
\hline 9 & 143.27 & 201.1 & 43.7 & 0.798 & 10.64 & 0.941 & -16.189 & 0.97 & 18.7 & 272.15 & 0.881 & 1.14 \\
\hline 12 & 185.35 & 258.6 & 49.6 & 0.806 & 10.63 & 0.822 & -29.636 & 1.384 & 24.3 & 300.15 & 0.89 & 1.19 \\
\hline 14 & 213.403 & 291.9 & 53.1 & 0.81 & 10.62 & 0.739 & -38.601 & 1.535 & 27.9 & 313.15 & 0.888 & 1.26 \\
\hline 16 & 241.456 & 321.8 & 56.4 & 0.813 & 10.61 & 0.690 & -47.565 & 1.693 & 31.6 & 319.95 & 0.88 & 1.26 \\
\hline 18 & 269.509 & 348.9 & 59.3 & 0.862 & 10.60 & 0.648 & -56.530 & 1.86 & 35.3 & 326.05 & 0.88 & 1.29 \\
\hline
\end{tabular}




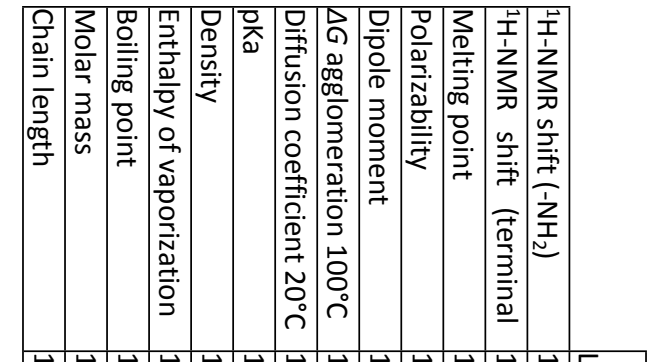

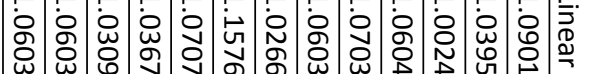

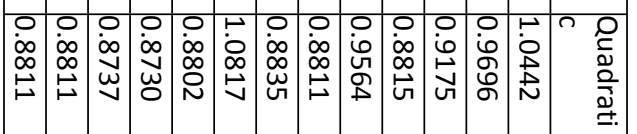

이일

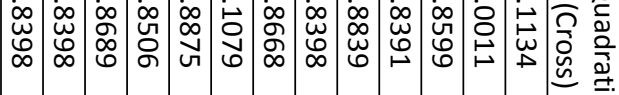

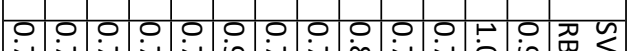

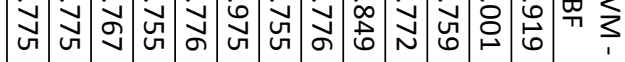

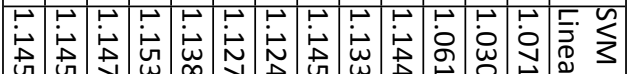

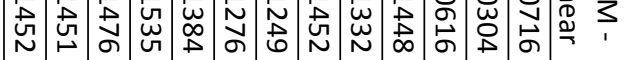

官

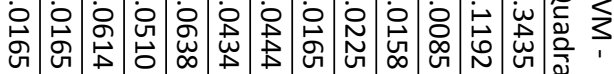

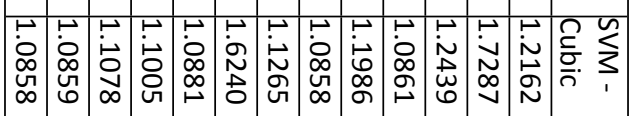

官客

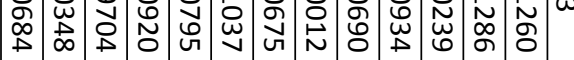

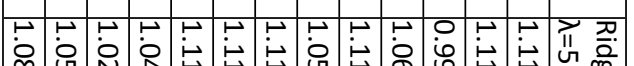

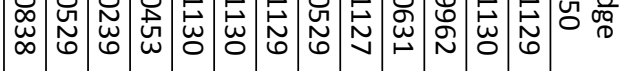

家穴

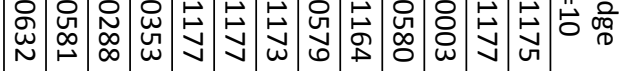

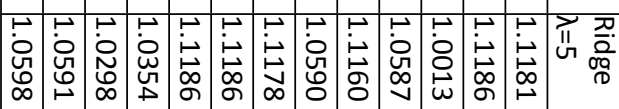

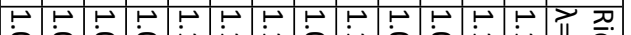

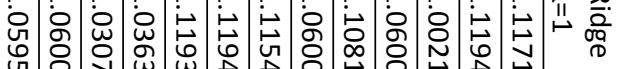

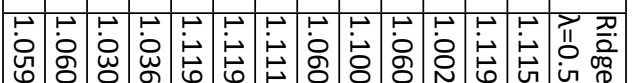

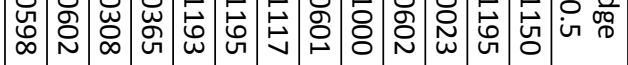

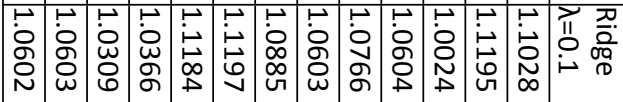

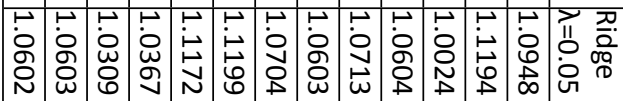

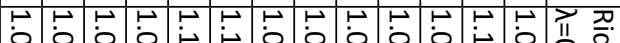

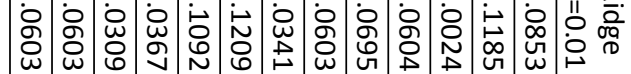

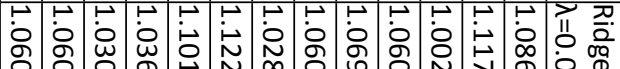

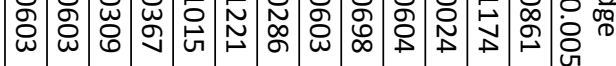

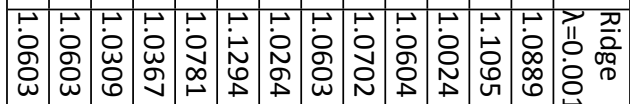

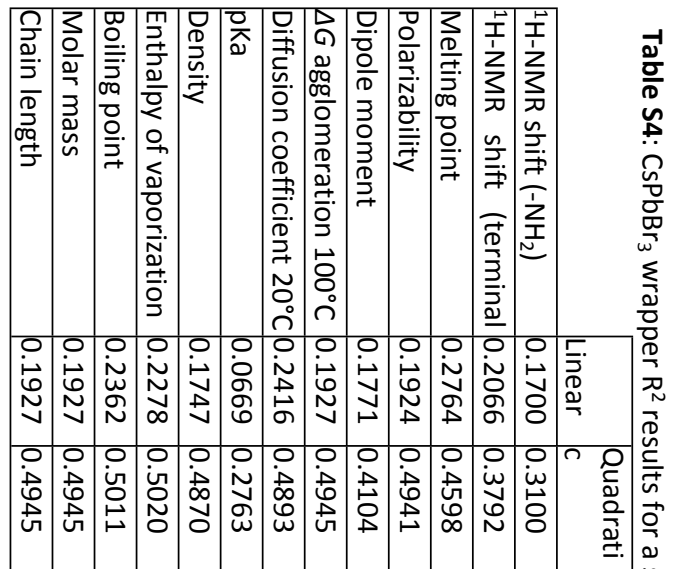

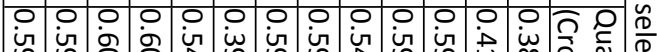
告

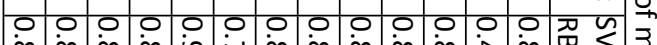

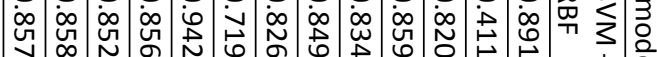
ᄀ

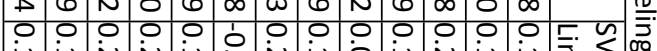

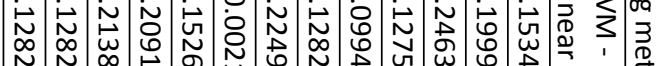

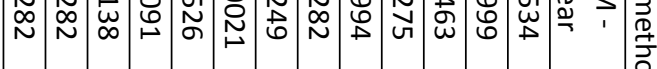

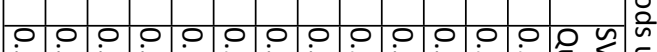

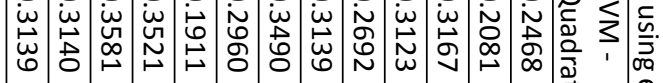

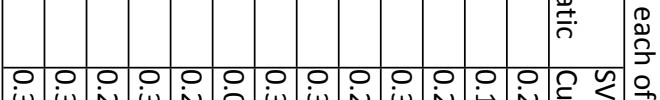

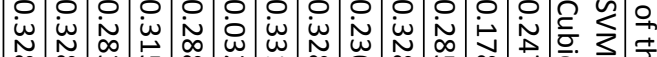

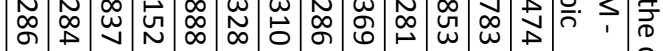
이일

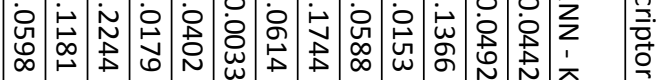

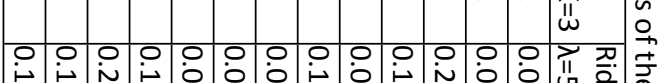

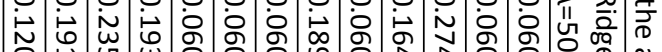

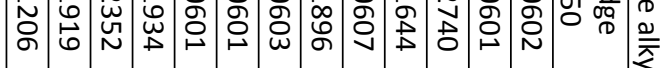
일

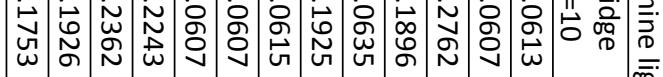
일 客

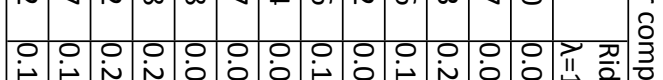

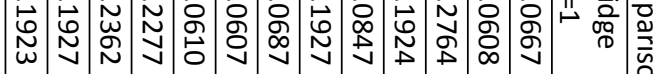

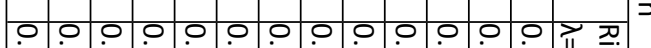

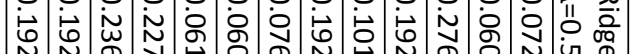

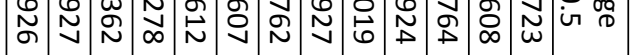

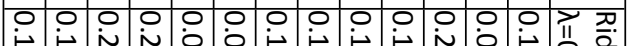

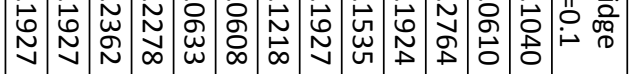

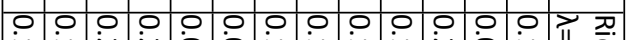

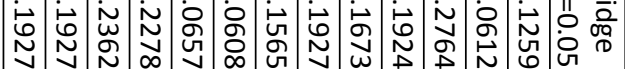

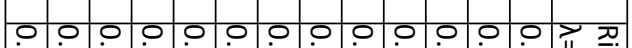

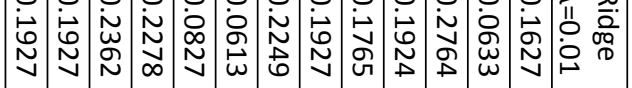

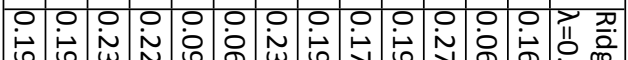

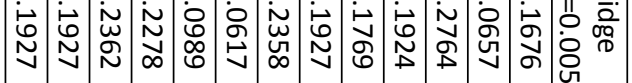

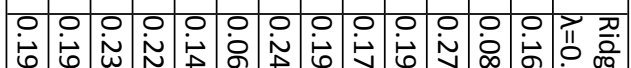

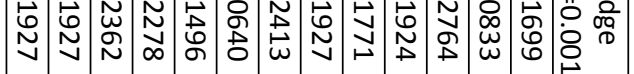


Table S6. Deconvolution of PL emission spectra for a validation set of six samples. Multi-peak Gaussian fitting has been used to determine the fractional populations for different layer thicknesses $(n=1,2$, $3,4,5,6$, and bulk).

\begin{tabular}{|c|c|c|c|c|c|c|c|c|c|c|c|}
\hline Entry & $\begin{array}{c}\text { Chain- } \\
\text { length } \\
\left(\mathrm{C}_{n}\right)\end{array}$ & $\begin{array}{c}T \\
\left({ }^{\circ} \mathrm{C}\right)\end{array}$ & $\begin{array}{c}\text { Conc. } \\
(x)\end{array}$ & $n=2$ & $n=3$ & $n=4$ & $n=5$ & $n=6$ & $\begin{array}{c}n= \\
\text { bulk }\end{array}$ & $n_{\text {avg. }}$ & $\begin{array}{c}\text { TEM } \\
\text { thicknes } \\
\mathrm{s}(\mathrm{nm})\end{array}$ \\
\hline 1 & 6 & 70 & $1: 15$ & - & 0.030 & 0.101 & 0.135 & 0.334 & 0.400 & 7.2 & 4.0 \\
\hline 2 & 7 & 90 & $1: 15$ & - & 0.128 & 0.175 & 0.195 & 0.166 & 0.336 & 6.4 & 3.7 \\
\hline 3 & 7 & 70 & $1: 30$ & 0.009 & 0.392 & 0.279 & 0.185 & 0.068 & 0.067 & 4.3 & 2.5 \\
\hline 4 & 18 & 80 & $1: 25$ & 0.059 & 0.246 & 0.306 & 0.119 & 0.187 & 0.083 & 4.6 & 2.9 \\
\hline 5 & 16 & 90 & $1: 25$ & 0.060 & 0.562 & 0.192 & 0.075 & 0.083 & 0.027 & 3.7 & 2.3 \\
\hline 6 & 16 & 90 & $1: 40$ & - & 0.413 & 0.328 & 0.068 & 0.048 & 0.142 & 4.6 & 2.8 \\
\hline
\end{tabular}

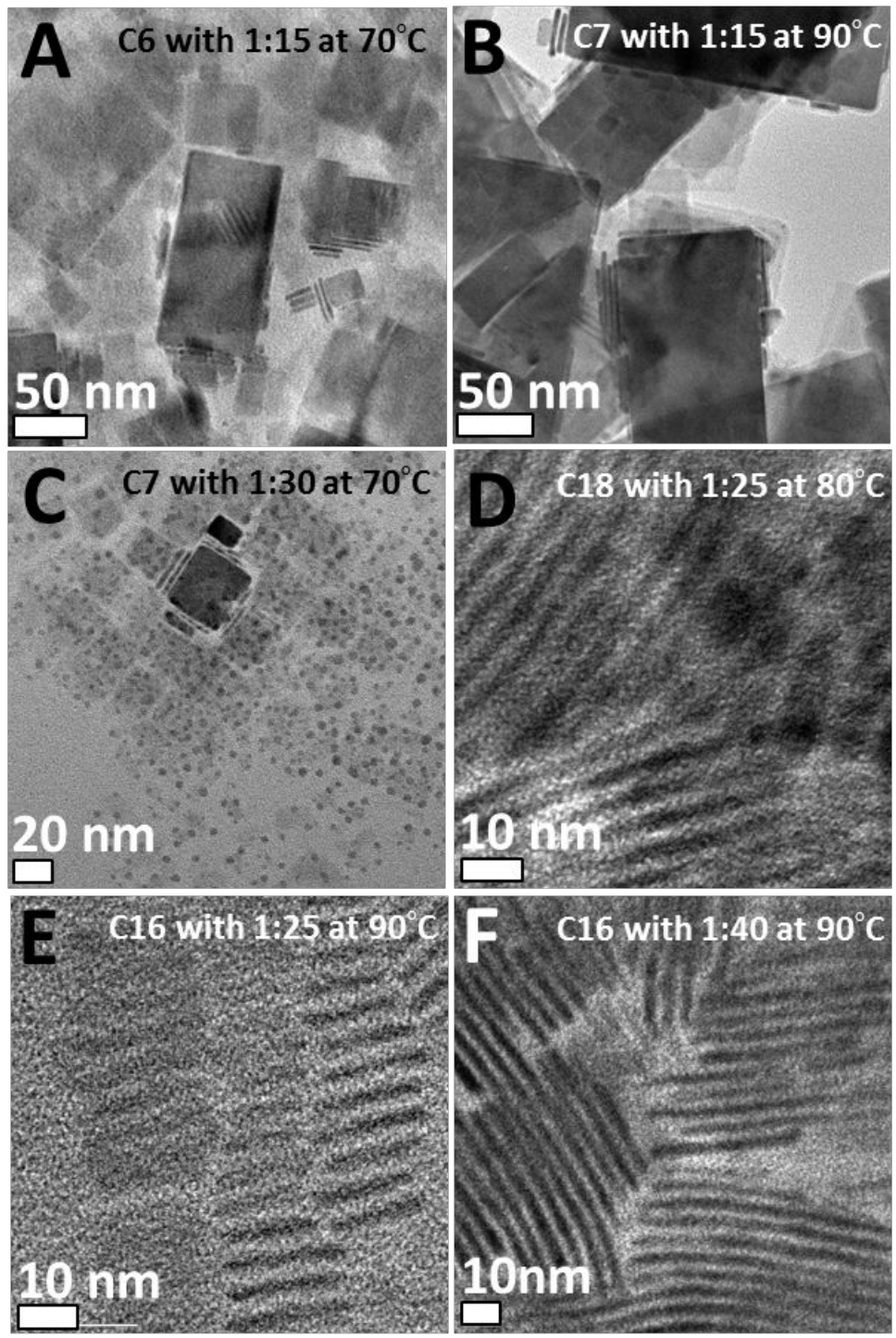

Figure S5. TEM images of $\mathrm{CsPbBr}_{3}$ nanoplatelets corresponding to the validation set stabilized at different reaction conditions: A) C6 with 1: $x=1: 15$ at $70^{\circ} \mathrm{C}$; B) C7 with 1:x $=1: 15$ at $90^{\circ} \mathrm{C}$; C) C7 with $1: x=1: 30$ at $\left.70^{\circ} \mathrm{C} ; \mathrm{D}\right) \mathrm{C} 18$ with $1: x=1: 25$ at $80^{\circ} \mathrm{C}$; E) C16 with $1: x=1: 25$ at $90^{\circ} \mathrm{C}$; and F) $\mathrm{C} 16$ with $1: x=$ $1: 40$ at $90^{\circ} \mathrm{C}$. 
Table S7.

Deconvolution of PL emission spectra for samples predicted to be have an $n_{\text {avg }}$ of 3.0. Multi-peak Gaussian fitting has been used to determine the fractional populations for different layer thicknesses ( $n=1,2,3,4,5,6$, and bulk).

\begin{tabular}{|c|c|c|c|c|c|c|c|c|c|c|}
\hline Entry & $\begin{array}{c}\text { Alkylamine } \\
\text { Chain- } \\
\text { length }\left(\mathrm{C}_{n}\right)\end{array}$ & $\begin{array}{c}T \\
\left({ }^{\circ} \mathrm{C}\right)\end{array}$ & Conc. $(\mathrm{x})$ & $n=2$ & $n=3$ & $n=4$ & $n=5$ & $n=6$ & $\begin{array}{c}n= \\
\text { bulk }\end{array}$ & $n_{\text {avg. }}$ \\
\hline 1 & 9 & 104 & $1: 32$ & 0.318 & 0.514 & 0.131 & 0.023 & 0.015 & 0 & 2.90 \\
\hline 2 & 12 & 50 & $1: 30$ & 0.446 & 0.439 & 0.065 & 0.033 & 0.017 & 0 & 2.74 \\
\hline 3 & 14 & 82 & $1: 28$ & 0.642 & 0.286 & 0.053 & 0.018 & 0 & 0 & 2.45 \\
\hline
\end{tabular}
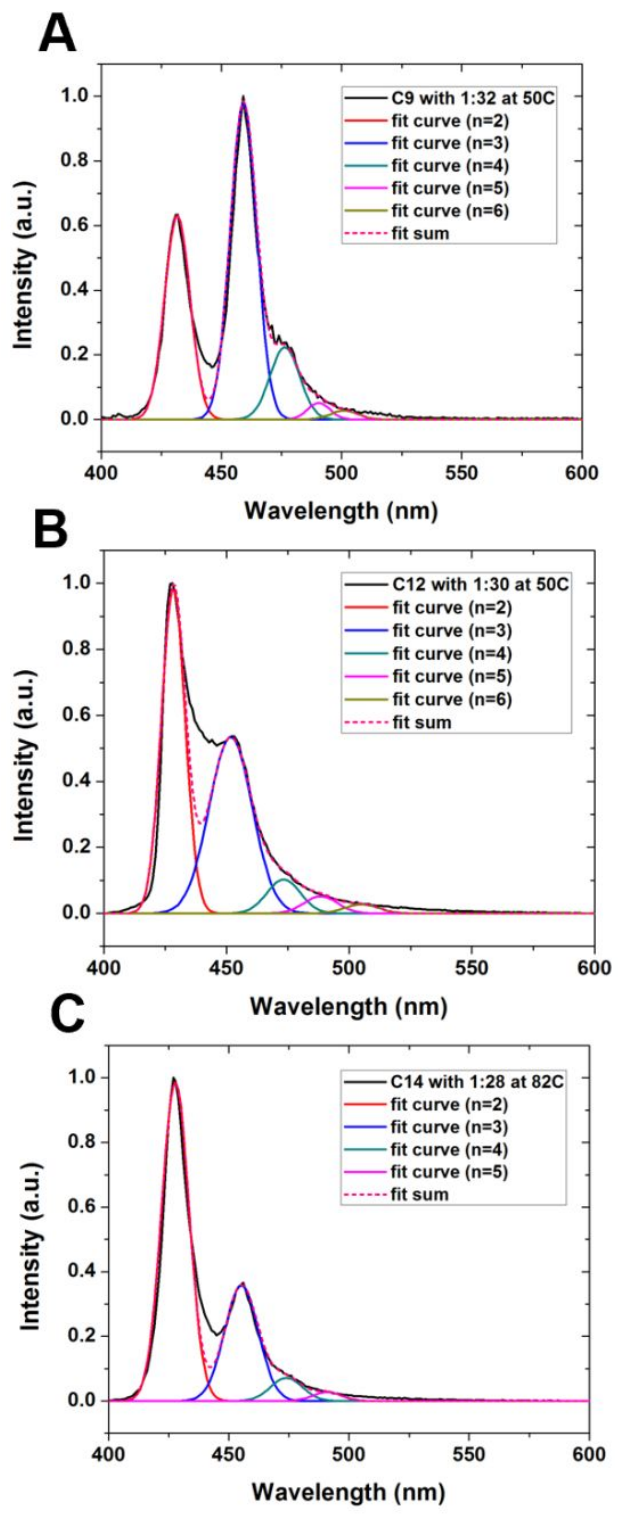

Figure S6: PL emission spectra and Gaussian peak fits for three samples predicted to have an $n_{\text {avg. }}$ of 3.0; $A, B$, and $C$ correspond to entries 1,2 , and 3 in Table S7, respectively. 


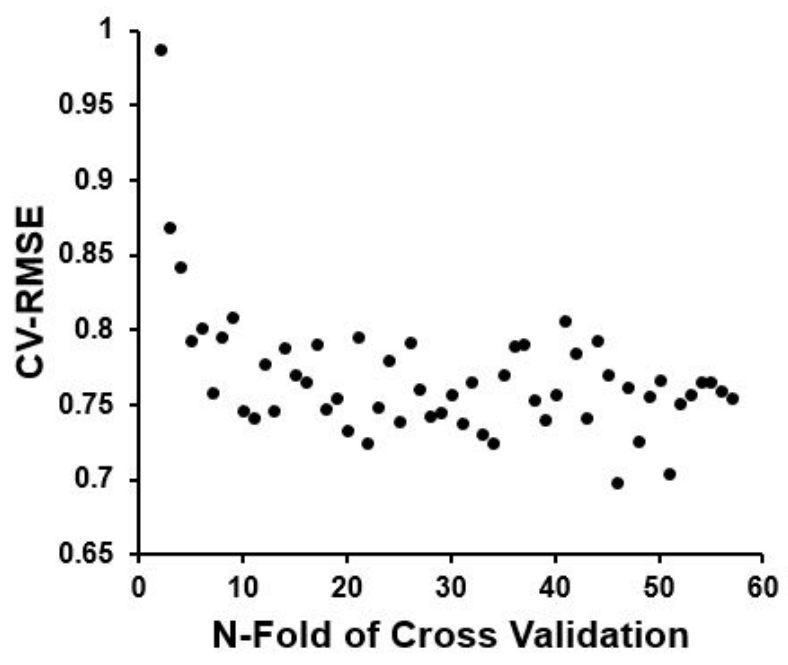

Figure S7. Plot of cross-validation error versus the number of folds in cross validation for the SVM model with a radial basis function kernel using diffusion coefficient as the alkylamine descriptor.

\section{References}

(1) Haynes, William M., David R. Lide, and T. J. B. CRC Handbook of Chemistry and Physics : A Ready-Reference Book of Chemical and Physical Data, 97th ed.; Boca Raton, Florida, 2017.

(2) ACD/Percepta. Advanced Chemistry Development Inc.: Toronto, ON 2017.

(3) Cho, J.; Choi, Y. H.; O'Loughlin, T. E.; De Jesus, L.; Banerjee, S. Ligand-Mediated Modulation of Layer Thicknesses of Perovskite Methylammonium Lead Bromide Nanoplatelets. Chem. Mater. 2016, 28 (19), 6909-6916.

(4) Matulis, D.; Bloomfield, V. A. Thermodynamics of the Hydrophobic Effect. II. Calorimetric Measurement of Enthalpy, Entropy, and Heat Capacity of Aggregation of Alkylamines and Long Aliphatic Chains. Biophys. Chem. 2001, 93 (1), 53-65.

(5) Hanwell, M. D.; Curtis, D. E.; Lonie, D. C.; Vandermeerschd, T.; Zurek, E.; Hutchison, G. R. Avogadro: An Advanced Semantic Chemical Editor, Visualization, and Analysis Platform. J. Cheminform. 2012, 4 (17), 1-17.

(6) National Institute of Advanced Industrial Science and Technology, Spectral Database for Organic Compounds. http://sdbs.db.aist.go.jp (accessed July 25, 2017) 